OPEN ACCESS

Edited by:

Zhengli Shi,

Chinese Academy of Sciences,

China

Reviewed by:

Yulan Liu,

Wuhan Polytechnic University,

China

Daniel C. Pevear,

VenatoRx Pharmaceuticals,

United States

*Correspondence:

Zili Li

lizili@mail.hzau.edu.cn

Specialty section:

This article was submitted to

Virology,

a section of the journa

Frontiers in Microbiology

Received: 28 June 2019 Accepted: 18 September 2019

Published: 04 October 2019

Citation:

Qian S, Zhang W, Jia X, Sun Z,

Zhang Y, Xiao Y and Li Z (2019) Isolation and Identification of Porcine Epidemic Diarrhea Virus and Its Effect on Host Natural Immune Response.

Front. Microbiol. 10:2272.

doi: 10.3389/fmicb.2019.02272

\section{Isolation and Identification of Porcine Epidemic Diarrhea Virus and Its Effect on Host Natural Immune Response}

\author{
Shaoju Qian', Weida Zhang ${ }^{1}$, Xiangchao Jia', Zhijian Sun', Yang Zhang ${ }^{1}$, \\ Yuncai Xiao ${ }^{1,2,3}$ and Zili $\mathrm{Li}^{1,2,3 *}$ \\ 'State Key Laboratory of Agricultural Microbiology, Department of Preventive Veterinary Medicine, College of Veterinary \\ Medicine, Huazhong Agricultural University, Wuhan, China, ${ }^{2}$ Key Laboratory of Preventive Veterinary Medicine in Hubei \\ Province, The Cooperative Innovation Center for Sustainable Pig Production, Wuhan, China, ${ }^{3}$ Key Laboratory of \\ Development of Veterinary Diagnostic Products, Ministry of Agriculture of the People's Republic of China, Wuhan, China
}

Porcine epidemic diarrhea (PED) is a highly infectious intestinal disease caused by porcine epidemic diarrhea virus (PEDV). A PEDV strain was isolated from the piglet intestinal tract in Vero cells in Jiangsu Province, designated as the JS-A strain. PEDV was identified as the isolated virus by cytopathology, immunofluorescence assay, western blotting, transmission electron microscopy, and sequence analysis. The full-length genome of the JS-A isolate and the S gene were systematically analyzed, indicating that PEDV JS-A belongs to the G2a subtype, which is closely related to the prevalent PEDV in many countries and different from many current vaccines. Animal regression tests showed that piglets that are orally infected with the virus continue to develop diarrhea with yellowish and unpleasant odors. Further, piglets showed reduced food consumption and weight loss in the challenged group, while there were no abnormalities in the control group. In addition, Toll-like receptors (TLRs), RIG-I, and the downstream medium gene in the intestinal mucosa of newborn pigs infected with PEDV JS-A strain were studied. The neonatal Fc receptor (FcRn) was the only lgG transport receptor and protected lgG from degradation. Therefore, PEDV JS-A infection might inhibit FcRn expression by downregulating TLRs and downstream signaling molecules. Taken together, isolation of the JS-A variant contributes to evolutionary analysis of the diarrhea virus. Further, the experimental infection model lays a foundation for further research related to vaccine development and the antiviral natural immune response of infected piglets, which helps us to better understand PEDV pathogenesis and immune mechanism.

Keywords: porcine epidemic diarrhea virus, neonatal fc receptor, Toll-like receptor, NF-кB, neutralizing antibody

\section{INTRODUCTION}

Porcine epidemic diarrhea virus (PEDV) causes severe enteritis, watery diarrhea, vomiting, dehydration, and even death in suckling piglets. In the 1970s, the first PEDV outbreak occurred in Europe (Wood, 1977). Subsequently, the virus affected many countries such as the Czech Republic, Belgium, Hungary, South Korea, Italy, and Thailand (Song and Park, 2012). Since 2010, a 
large-scale outbreak of PEDV has occurred in swine herds around China. Then, the emergence of PEDV occurred in Ohio in the United States in 2013, which spread throughout the United States (Huang et al., 2013). Currently, due to lack of effective vaccines, isolation and identification of new PEDV strains to develop appropriate vaccines for prevention and control of the disease have garnered great interest.

PEDV belongs to the genus Alphacoronavirus within the Coronaviridae family (Brian and Baric, 2005). Its genome size is about $28 \mathrm{~kb}$, and it possesses at least seven open reading frames (ORF): ORF1a, ORF1b, spike (S), ORF3, envelope (E), membrane $(\mathrm{M})$, and nucleocapsid $(\mathrm{N})$. PEDV $\mathrm{S}$ protein plays a critical role in inducing neutralization antibodies, specific receptor binding, and membrane fusion. In addition, the $S$ gene has shown a high degree of genetic variability. Therefore, it is often used to analyze PEDV genetic variation. According to phylogenetic analysis of $\mathrm{S}$ genes, PEDV strains have been divided into two clusters: genome I subtype and genome II subtype (Lee et al., 2010; Li et al., 2013; Chen et al., 2014). Further, several variant PEDV strains have been identified, which were characterized by insertion and deletion (INDEL) in labeled (S) genes named S-INDEL PEDV. The pathogenesis of PEDV is strain specific. Studies on its pathogenesis in piglets suggest that the PEDV non-S-INDEL strain has stronger pathogenicity compared to the PEDV S-INDEL strain (Chen et al., 2014; Yamamoto et al., 2015; Wang et al., 2016). PEDV JS-A belongs to the non-S-INDEL strain.

Upon viral infection, the host innate immune response is the first line of defense (Moynagh, 2005). NF- $\kappa B$ regulates expression of numerous components of the immune system, which is thought to be the hallmark of most infection, including coronaviruses (DeDiego et al., 2014). RNA viruses interact with pattern-recognition receptors (PRRs), including Toll-like receptors (TLRs) and RIG-I-like receptors (RLRs). Several in vitro studies have been conducted to clarify the role of PEDV in innate immune responses at the cellular level. Several other studies have determined that PEDV nucleocapsid protein $(\mathrm{N})$ inhibits the production of type I and III interferons (IFNs) (Ding et al., 2014; Cao et al., 2015a; Shan et al., 2018). PEDV non-S-INDEL infection inhibits the induction of proinflammatory cytokines and IFN-I by down-regulating TLRs and downstream signaling molecules (Temeeyasen et al., 2018).

Neonatal Fc receptor (FcRn) has been fully characterized to transfer maternal IgG to the fetus or newborn and protect IgG from degradation. In addition to IgG, FcRn binds to albumin, which regulates liver damage (Pyzik et al., 2017). Transmissible gastroenteritis virus (TGEV) infection up-regulates $\mathrm{pFcRn}$ expression and activates the NF- $\mathrm{BB}$ signaling pathway in IPEC-J2 cells (Guo et al., 2016a). In our study, the pathogenicity of JS-A in 5-day-old piglets was studied by clinical evaluation, quantitative analysis of virus copy number in the feces, histology, and immunohistochemistry. We also studied the gene regulation of TLRs, RIG-I, and downstream signaling pathways in the intestinal mucosa of pigs newly infected with JS-A. The purpose of this study was to investigate the genomic characteristics and pathogenicity of JS-A and the expression of TLRs, RIG-I, $\mathrm{NF}-\kappa \mathrm{B}$, and FcRn in the intestinal mucosa of infected piglets.

\section{MATERIALS AND METHODS}

\section{Clinical Samples and Virus Isolation}

Fecal samples and intestinal contents from piglets suffering from severe watery diarrhea were collected in a pig farm from Jiangsu Province. PEDV-positive samples were detected by realtime polymerase chain reaction (RT-PCR), based on the $\mathrm{N}$ gene, as previously described (Lee et al., 2015). Clinical samples were homogenized in DMEM (HyClone, Logan, UT, USA), centrifuged at 4,000 $\mathrm{rpm} / \mathrm{min}$, and filtered through a $0.22-\mu \mathrm{m}$ filter (Millipore, MA).

Vero (ATCC, CCL-81) cells were obtained from the China Center for Type Culture Collection (Wuhan, China) and were cultured in DMEM containing 10\% fetal bovine serum (FBS, Gibco, Waltham, MA, USA). Vero cells were incubated with $1 \mathrm{~mL}$ of filtered inoculum for $2 \mathrm{~h}$. After virus adsorption, cells were maintained in DMEM containing $8 \mu \mathrm{g} / \mathrm{mL}$ trypsin (Gibco, USA) and antibiotics. After $2 \mathrm{~h}$, the cells were maintained in DMEM containing $8 \mu \mathrm{g} / \mathrm{mL}$ trypsin at $37^{\circ} \mathrm{C}$ in $5 \% \mathrm{CO}_{2}$ until a cytopathic effect became visible. The supernatant was collected after the cytopathic effect was observed. The virus was cloned and purified by plaque purification three times and tested by $\mathrm{TCID}_{50}$.

\section{Immunofluorescence Assay}

At $24 \mathrm{~h}$ post-infection, cells were fixed with $4 \%$ paraformaldehyde in PBS for $15 \mathrm{~min}$ and permeabilized with $0.1 \%$ Triton X-100 for $10 \mathrm{~min}$. Subsequently, cells were incubated with PBST containing 5\% skim milk for $1 \mathrm{~h}$, followed by primary (PEDV polyclonal antibody; prepared in our laboratory) and secondary [fluorescein isothiocyanate (FITC)-labeled goat anti-rabbit IgG; ABclonal, China] antibodies for $1 \mathrm{~h}$. Finally, the samples were stained with DAPI for $15 \mathrm{~min}$ and examined using a fluorescence microscope (Olympus IX73, Japan).

\section{Western Blot}

At $24 \mathrm{~h}$ post-infection, cells lysates were prepared for $12 \%$ SDS-PAGE, and proteins were electroblotted onto a polyvinylidene difluoride membrane (Bio-Rad, USA). PEDV-N polyclonal antibody (prepared in our laboratory) was used as a primary antibody, followed by HRP-conjugated goat anti-rabbit IgG or anti-mouse IgG (ABclonal, China) as secondary antibodies. Proteins were visualized as described previously (Guo et al., 2016b).

\section{Electron Microscopy}

PEDV-infected Vero cells were examined by electron microscopy (EM), following previously described procedures (Chen et al., 2014). These samples were negatively stained with $2 \%$ sodium phosphotungstic acid ( $\mathrm{pH}$ 6.8) and examined using a Hitachi Model H-7650 TEM (Hitachi H-7000FA, Japan).

\section{Phylogenetic Analysis}

Total RNAs were extracted using TRIzol ${ }^{\circledR}$ reagent (Invitrogen, Carlsbad, CA, USA), and total RNA was reverse transcribed into cDNA using reverse transcriptase (TaKaRa, Osaka, Japan). The whole genome of JS-A was sequenced using 25 pairs of primers previously reported in Table $\mathbf{1}$ by Chen (Chen et al., 2012). 
Phylogenetic trees with 25 strains (Table 2) full-length genome and S-nucleotide sequences were generated using the distancebased adjacency method in MEGA7.

\section{Inoculation of Piglets With Porcine Epidemic Diarrhea Virus Strain JS-A}

This study was implemented according to the Guide of the Scientific Ethics Committee of Huazhong Agricultural University (HZAUSW-2018-011). Twelve 5-day-old piglets were purchased from a commercial pig farm, both PEDV RNA and antibody negative, and were randomly divided into two groups (6 piglets/ group) in two separate rooms. Piglets were fed a mixture of liquid milk replacer and had free access to water. After 1 day of adaptation, piglets in the challenged group received $3 \times 10^{5}$ $\mathrm{TCID}_{50}$ PEDV JS-A orally, while piglets in the control group received $3 \mathrm{~mL}$ maintenance medium orally. The piglets were observed daily for signs of vomiting, diarrhea, lethargy, weight, and body temperature until the end of the experiment. Intestinal segments, including the jejunum and ileum, were collected after euthanasia of three of six piglets in each group, selected at random, at $4 \mathrm{dpi}$ for histopathological and immunohistochemical examinations using the method described previously (Lee et al., 2010). At the end of the experiment ( $21 \mathrm{dpi})$, the remaining piglets were euthanized. Expressions of mRNA for TLRs, NF- $\mathrm{B}$

TABLE 1 | Primers for amplifications of the PEDV genomic fragments by RT-PCR.

\begin{tabular}{|c|c|c|c|}
\hline Name & Sequence $\left(5^{\prime}\right.$ to $\left.3^{\prime}\right)$ & Position in PEDV genome & Size (bp) \\
\hline PP1F & GTGGAATTCATTAGGTITG & $123-142$ & 1,380 \\
\hline PP1R & AAGCTTACGTATGAACCAAG & $1502-1483$ & \\
\hline PP2F & TGCTGGTCATGTTGTTGTTG & $1421-1440$ & 1,488 \\
\hline PP2R & TAGAAAGCGAAGCCATCAAC & 2908-2889 & \\
\hline PP3F & ATTGAAACTTCTITGTGGA & $2817-2836$ & 1,451 \\
\hline PP3R & TTCTCATTTGCAGCATTAAC & $4267-4248$ & \\
\hline PP4F & СTGCTCTCTCCTTGGATTCT & 3940-3959 & 1,478 \\
\hline PP4R & AGTATGGTCTAGCATGTGGA & $5417-5398$ & \\
\hline PP5F & TGTCACAGACAACAAACCTG & $5300-5319$ & 1,487 \\
\hline PP5R & CATCACCAAAGACATCAAAA & $6786-6767$ & \\
\hline PP6F & ATTGGTAATGTGATGCCTाT & $6609-6628$ & 1,323 \\
\hline PP6R & AAAGCTTAGTGCAAAGAAAG & $7931-7912$ & \\
\hline PP7F & TाTCAAAGGTTAAGAAATTCT & $7861-7881$ & 1,408 \\
\hline PP7R & CTCACAGTGGGTGGTGTGTAT & $9268-9248$ & \\
\hline PP8F & CGTTATACACACCACCCACT & $9244-9263$ & 1,304 \\
\hline PP8R & GGTAACAACAAAGCACACAA & $10547-10528$ & \\
\hline PP9F & CAGAGCATITGATTACCAT & $10465-10484$ & 1,470 \\
\hline PP9R & CAACTATGCCATCTCCTTCT & $11934-11915$ & \\
\hline PP10F & GGTGTGAGCGTATTGTTAAG & $11842-11861$ & 1,266 \\
\hline PP10R & AATGCATAGACACGATGAAT & $13107-13088$ & \\
\hline PP11F & TITGATTAAGGTAGGTGCTT & $13012-13031$ & 1,397 \\
\hline PP11R & CAACACCATCAATAAGGTTC & $14408-14389$ & \\
\hline PP12F & TATGGTGGTTGGGACAATAT & $14363-14382$ & 946 \\
\hline PP12R & CTTCCAAAAGTGTGACAGAA & $15308-15289$ & \\
\hline PP13F & ATTGCTTGAACGTTATGTGT & $15142-15161$ & 1,676 \\
\hline PP13R & САTTACССТTGCAAAAGATC & $16817-16798$ & \\
\hline PP14F & TTAAGCCTGATGTCTTCTTG & $16674-16693$ & 1,691 \\
\hline PP14R & CCTACAGCGAGTATCAAAAC & $18364-18345$ & \\
\hline PP15F & TGGACATGTATCCAGAATIT & $18312-18331$ & 1,455 \\
\hline PP15R & GCATGGAATAACCACACTTC & $19766-19747$ & \\
\hline PP16F & AAAATGTGGAGGTGGATGTT & 19670-19689 & 1,362 \\
\hline PP16R & GGCCCAATGTITATTATCG & $21031-21012$ & \\
\hline S-1F & TAAGTTGCTAGTGCGTAAT & $20572-20590$ & 1,692 \\
\hline$S-1 R$ & GTCAACACAGAAAGAACTA & 22263-22245 & \\
\hline S-2F & TGAGTCATGAACAGCCAA & 22003-22020 & 1,511 \\
\hline$S-2 R$ & CTGGTTGCGCTGTAGAA & $23513-23497$ & \\
\hline S-3F & GCGTCTCTCATAGGTGGT & $23491-23508$ & 1,310 \\
\hline S-3R & GTCCAAGAAACATCACTG & $24801-24784$ & \\
\hline ORF3.EF & CGTGCAGTGATGTITCTTGGAC & $24780-24801$ & 895 \\
\hline ORF3·ER & TTATACGTCAATAACAGTACT & $25674-25654$ & \\
\hline EMF & CTTCACTTGTCACCGGTTGT & $25557-25576$ & 1,097 \\
\hline EMR & CCTCAGTACGAGTCCTATAAC & $26549-26529$ & \\
\hline MF & GGCGAATTCAATATGTCTAACGGTTC & $25670-25695$ & 675 \\
\hline $\mathrm{MR}$ & CGCGTCGACCCATAAAGTTCTGTT & $26368-26362$ & \\
\hline NF & GGATCCATGGCTTCTGTCAGCTाT & $26107-26218$ & 1,332 \\
\hline NR & GACGTCACATTGTTAATITCCTGTACC & & \\
\hline $3 F$ & GGAAAAGAAGAACAAGCGT & $27355-27373$ & 580 \\
\hline $3 R$ & 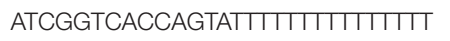 & & \\
\hline
\end{tabular}


and FcRn on piglet intestinal mucosa in each group were detected by RT-qPCR at 4 and $21 \mathrm{dpi}$.

\section{Real-Time Polymerase Chain Reaction}

Viral RNA of PEDV in piglet feces or intestinal mucosa was detected as previously described (Subramaniam et al., 2018).
The specific porcine primers are referenced in previous publications in Table 3 (Guo et al., 2016a; Temeeyasen et al., 2018) were synthesized by the GenScript Company (Nanjing, China). The $\mathrm{N}$ gene amplified from the PEDV JS-A strain was cloned into pMD18-T (Takara, Dalian). The known plasmid concentrations were serially diluted 10 -fold to generate a standard

TABLE 2 | Comparison of nucleotide sequences of the JS-A isolate with other strains.

\begin{tabular}{|c|c|c|c|c|c|}
\hline Strains & Countries & Accession & Strains & Countries & Accession \\
\hline JS-A & China & MH748550 & MEX/124/2014 & USA & KJ645700 \\
\hline LZC & China & EF185992 & $\mathrm{MN}$ & USA & KF468752 \\
\hline AJ1102 & China & JX188454 & PC21A & USA & KR078299 \\
\hline $\mathrm{AH} 2012$ & China & KC210145 & USA/Colorado/2013 & USA & KF272920 \\
\hline GD-B & China & JX088695 & CH/JX-2/2013 & China & KJ526096 \\
\hline $\mathrm{CH}-\mathrm{S}$ & China & JN547228 & $\mathrm{CH} / \mathrm{JX}-1 / 2013$ & China & KF760557 \\
\hline $\mathrm{IA} 2$ & USA & KF468754 & $\mathrm{CH} / \mathrm{HNAY} / 2015$ & China & KR809885 \\
\hline SM98 & Korea & GU937797 & LC & China & JX489155 \\
\hline Attenuated DR13 & Korea & JQ023162 & FL2013 & China & KP765609 \\
\hline CV777 & Switzerland & AF353511 & DR13 & Korea & JQ023162 \\
\hline YN144 & China & КT021232 & JS2008 & China & KC109141 \\
\hline JS-HZ2012 & China & KC210147 & Belgorod/dom/2008 & Russia & MF577027 \\
\hline GD-1 & China & JX647847 & & & \\
\hline
\end{tabular}

TABLE 3 | Primers used for RT-qPCR analysis of genes expression of pig intestinal mucosa.

\begin{tabular}{|c|c|c|c|}
\hline Gene name & Primer sequence $\left(5^{\prime}-3^{\prime}\right)$ & Amplicon length (bp) & Accession number \\
\hline GAPDH-F & GGAAAGGCCATCACCATCTT & \multirow[t]{3}{*}{85} & \multirow[t]{3}{*}{ XM_021091114.1 } \\
\hline GAPDH-R & CATGGTCGTGAAGACACCAG & & \\
\hline FCRn-F & GGCGACGAGCACCACTACTG & & \\
\hline FcRn-R & AGCCGACCATGATTCCAACC & \multirow{2}{*}{88} & \multirow{2}{*}{$\begin{array}{l}\text { HQ026019.1 } \\
\text { XM_005653576.3 }\end{array}$} \\
\hline TLR2-F & GAGTCTGCCACAACTCAAAGA & & \\
\hline TLR2-R & CAGAACTGACAACATGGGTAGAA & \multirow{2}{*}{72} & \multirow{2}{*}{ KT735340.1 } \\
\hline TLR3-F & GCGGTCCTGTTCAGTTCT & & \\
\hline TLR3-R & AAGGCATCTGCTGGGATाT & \multirow{2}{*}{74} & \multirow{2}{*}{ AB078418.1 } \\
\hline TLR4-F & AACTGCAGGTGCTGGATTTAT & & \\
\hline TLR4-R & CCGTCAGTATCAAGGTGGAAAG & \multirow{2}{*}{77} & \multirow{2}{*}{ DQ647699.2 } \\
\hline TLR7-F & CCCAGGTCCTCGAATCATTAC & & \\
\hline TLR7-R & CATTAAGAGGCAAGGAGGAAGA & \multirow{2}{*}{77} & \multirow{2}{*}{ KF019635.1 } \\
\hline TLR8-F & CTTGATGATGACGCTGCTTC & & \\
\hline TLR8-R & GGTGTGTCACTCCTGCTATTC & \multirow{2}{*}{82} & \multirow{2}{*}{ KC860785.1 } \\
\hline TLR9-F & ССТСАСАСАТСТСТСАСТСАAG & & \\
\hline TLR9-R & GGTGACAATGTGGTTGTAGGA & \multirow{3}{*}{91} & \multirow{3}{*}{ KC011279.1 } \\
\hline RIG-I-F & GAGCCCTTGTGGATGCTITA & & \\
\hline RIG-I-R & GGGTCATCCCTATGTTCTGATTC & & \\
\hline TRIF-F & CTCCGGTGCAGTCAAACA & \multirow[t]{2}{*}{91} & \multirow[t]{2}{*}{ KC969185.1 } \\
\hline TRIF-R & GGTAGTGTGTGCTGGTITCT & & \\
\hline MyD88A-F & GGCAGCTGGAACAGACCAA & \multirow[t]{2}{*}{41} & \multirow[t]{2}{*}{ EU056736.1 } \\
\hline MyD88A-R & GGCAGGACATCTCGGTCAGA & & \\
\hline MyD88B-F & TGCAGGTGCCCATCAGAAG & \multirow[t]{2}{*}{64} & \multirow[t]{2}{*}{ EU056737.1 } \\
\hline MyD88B-R & TGATGAACCGCAGGATGCT & & \\
\hline$N F-\kappa B 1(p 105)-F$ & GAGGTGCATCTGACGTATTC & \multirow[t]{2}{*}{118} & \multirow[t]{2}{*}{ NM_001048232.1 } \\
\hline$N F-\kappa B 1(p 105)-R$ & CACATCTCCTGTCACTGCAT & & \\
\hline$N F-\kappa B 1(p 50)-F$ & AAGCACGGAACTGTAGACAC & \multirow[t]{2}{*}{107} & \multirow[t]{2}{*}{ KC316024.1 } \\
\hline$N F-\kappa B 1(p 50)-R$ & TCTGTGGTITCTGTGACTITCC & & \\
\hline RELA-F & ACATGGACTTCTCAGCCCTTCTGA & \multirow[t]{2}{*}{285} & \multirow[t]{2}{*}{ CN155798.1 } \\
\hline RELA-R & CCGAAGACATCACCCAAAGATGCT & & \\
\hline TRAF6-F & GGGAACGATACGCCTTACAA & \multirow[t]{2}{*}{156} & \multirow{2}{*}{ NM_001105286.1 } \\
\hline TRAF6-R & СTCTGTCTTAGGGCGTCC & & \\
\hline
\end{tabular}


curve in each plate. The amount of PEDV viral RNA in the test sample was calculated based on the cycle curve threshold (Ct) value of the standard curve. Expression levels of genes were calculated relative to the expression of GAPDH using the delta-delta cycles to threshold $\left(2^{-\Delta \Delta C t}\right)$ method.

\section{IgG/lgA Enzyme-Linked Immunosorbent Assay and Neutralization Assay}

The PEDV-specific IgG/IgA antibodies were measured in serum samples by ELISA as previously described (Subramaniam et al., 2018). Polystyrene 96-well microplates were coated with purified PEDV $\mathrm{N}$ diluted in PBS containing Tween-20 (PBST) and incubated overnight at $4^{\circ} \mathrm{C}$, followed by the removal of the coating solution. The plates were blocked with $100 \mu \mathrm{L} 5 \%$ skimmed milk in PBST and incubated at $37^{\circ} \mathrm{C}$ for $30 \mathrm{~min}$. After which, $100 \mu \mathrm{L}$ of serum samples (diluted 1:200) per well was added and incubated at $37^{\circ} \mathrm{C}$ for $1 \mathrm{~h}$, respectively. After washing the plates, HRP-conjugated goat anti-swine IgG or goat anti-swine IgA was incubated for $1 \mathrm{~h}$. The plates were washed and incubated with TMB solution (Sigma-Aldrich) for $10 \mathrm{~min}$. The absorbance was measured at $450 \mathrm{~nm}$ with a termination solution (2 $\mathrm{M} \mathrm{H}_{2} \mathrm{SO}_{4}$ ).

PEDV isolate JS-A was used to determine the presence of neutralization (PEDV nucleocapsid protein) in serum samples as previously described (Oh et al., 2014). Vero cells were grown to $90 \%$ confluence in the 96 -well plates. Further, 200 TCID $_{50}$ of JS-A virus was diluted in DMEM in a $50 \mu \mathrm{L}$ volume. Subsequently, $50 \mu \mathrm{L}$ of 2 -fold serial dilutions of inactivated serum samples was added to the diluted virus and incubated at $37^{\circ} \mathrm{C}$ for $1 \mathrm{~h}$. The mixture was inoculated into Vero cells and incubated at $37^{\circ} \mathrm{C}$ for $1 \mathrm{~h}$. After removing the mixture and washing the plates, the virus in the maintenance medium was incubated in a $37^{\circ} \mathrm{C}$ and $5 \% \mathrm{CO}_{2}$ incubator for 2 days. The neutralization titer was calculated as the reciprocal of the highest serum dilution that neutralizes PEDV infection.

\section{Statistical Analysis}

Data were analyzed as mean \pm SEM. Differences among groups were performed by one-way ANOVA using GraphPad Prism software. The significance level for all analyses was set as ${ }^{*} p<0.05,{ }^{* *} p<0.01$ and ${ }^{* * *} p<0.001$.

\section{RESULTS}

\section{Virus Isolation and Characterization}

The treated samples were inoculated onto Vero cells. Cytopathic effects, including cell fusion, syncytium, and detachment, were observed at $36 \mathrm{~h}$ post-infection (hpi). No cytopathic effects were observed in the control wells (Figure 1A). To confirm the occurrence of virus multiplication, the infectivity of the JS-A strain (purified by plaque in Vero cells) was assessed by western blot (WB) and IFA using the PEDV $\mathrm{N}$ protein polyclonal antibody. IFA results demonstrated a green signal in infected Vero cells, but not in the mock-infected cells (Figure 1A).
Western blot analysis also detected $\mathrm{N}$ protein in PEDV-infected Vero cells (Figure 1B). According to the structural analysis of the infected cells by transmission electron microscopy, PEDV virus particles were round and showed obvious radial protrusion (Figure 1C), which is typical of coronaviruses. The plaquepurified virus was titrated to $10^{5.625} \mathrm{TCID}_{50} / \mathrm{mL}$.

\section{Phylogenetic Tree Analysis of JS-A Genomic Sequence}

The JS-A genome was sequenced and deposited in GenBank (MH748550). To further understand the evolutionary relationship between JS-A and other PEDV strains, a phylogenetic tree was constructed based on its genome and the $S$ gene sequence (Figure 2). These two phylogenetic trees all indicated that PEDV was mainly divided into two groups: group I consists mainly of classical strains and vaccine strains, while group II consists mainly of variant strains of China and mutant strains of the United States and South Korea. PEDV JS-A shared homology with the GD-1 strain of $99.9 \%$, belong to non-S INDEL strain. Homology with the classic strain CV777, LZC, and SM98 was 96.8, 96.5, and 96.6\%, respectively. The results showed that the $S$ gene had 15 base insertions at 20806-20817 nt and 21,046-21,048 nt, and nine base deletions at 21,114-21,119 nt and $24,225-24,227 \mathrm{nt}$. It is currently a prevalent species compared to the classical strain CV777.

\section{Clinical Observations in Pigs Challenged With JS-A}

To explore the pathogenicity of PEDV JS-A in piglets, we observed their clinical symptoms. All piglets from the control group were in great health throughout the study without observation of clinical symptoms. However, three of six piglets in the challenged group developed diarrhea at $1 \mathrm{dpi}$, and the additional three piglets developed various degrees of diarrhea, vomiting, lethargy, and anorexia at 2-6 dpi. Drowsiness, anorexia, and watery diarrhea developed most severely at 3-6 dpi, and piglets gradually recovered thereafter (Figure $\mathbf{3 A}$ ). No significant temperature change was observed between the two groups (Figure 3B). The infected group showed significant weight loss, while the uninfected piglets experienced weight gain (Figure 3C). Despite watery diarrhea with vomiting, drowsiness, and anorexia, no deaths occurred during the study period. These results indicate that JS-A is pathogenic to newborn pigs.

\section{Fecal Shedding, Virus Distribution, and Neutralizing Antibody Against JS-A}

To determine the fecal viral shedding, we attempted to detect viral RNA in rectal swab samples from 1 to 21 dpi by RT-PCR. Viral RNA was detected in 6/6 rectal swab samples collected from 1 to $14 \mathrm{dpi}$ in the challenged group. PEDV RNA copies reached the peak of $10^{4}-10^{7}$ copies $/ \mathrm{mL}$ in the homogenate rectal swab supernatant at 2-7 dpi, and the PEDV RNA was detected up to 14 dpi (Figure 4A). Notably, PEDV RNA was not detected in piglet samples from negative controls throughout the study. 


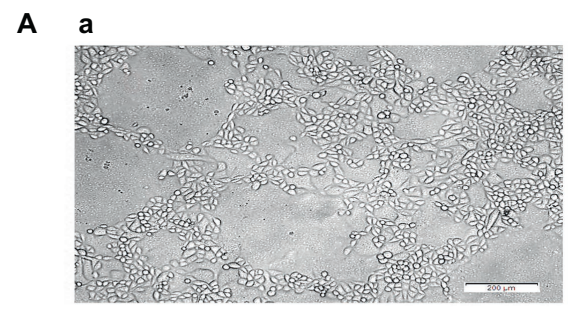

c

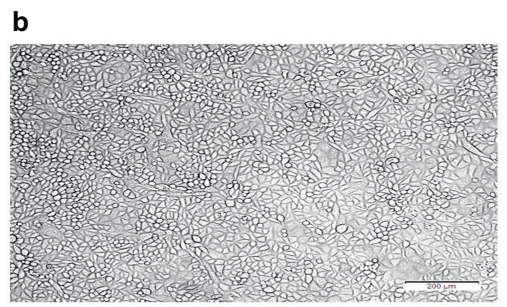

d

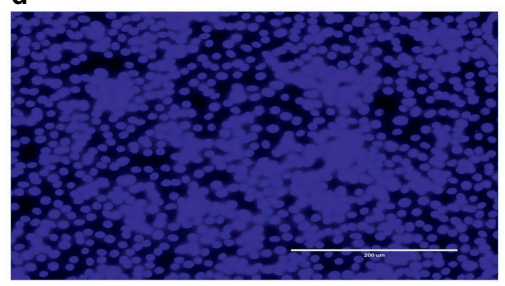

2

B
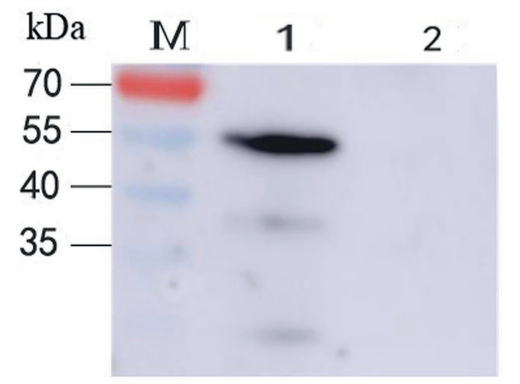

C

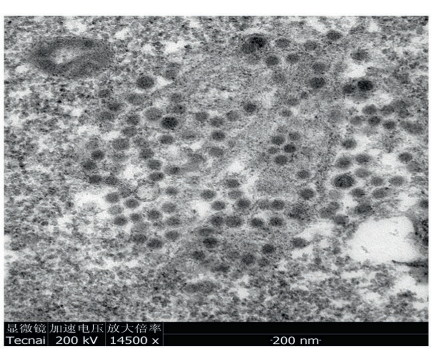

FIGURE 1 | The isolated PEDV strain JS-A was identified. (A) Simulated infected Vero cells of 24 hpi (a) and negative control Vero cells at 24 hpi (b). PEDVinfected (c) or negative control Vero cells (d) were examined by IFA at 24 hpi using polyclonal antibodies against PEDV N protein. (B) PEDV-infected or negative control Vero cells were subjected to analysis at 24 hpi with polyclonal antibodies against PEDV N protein by western blot; (C) electron microscopic images of purified PEDV particles were analyzed.
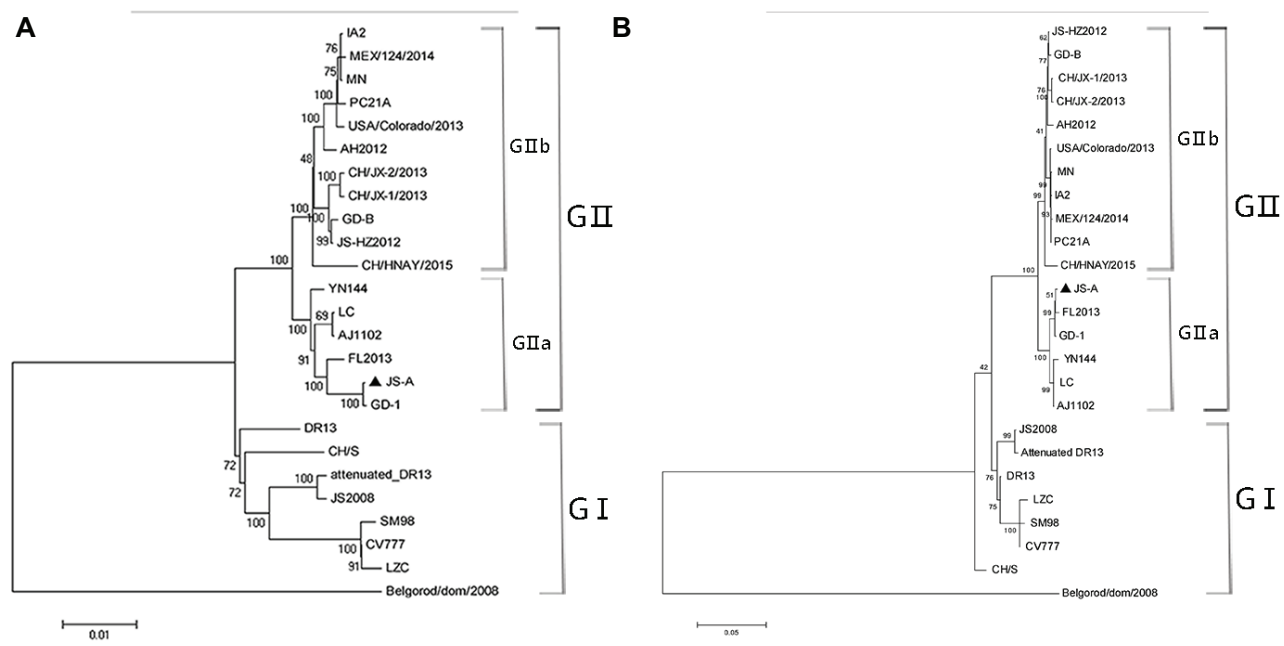

FIGURE 2 | Phylogenetic analysis was performed using the nucleotide sequences of the complete genome (A) and S genes (B) of the PEDV strain from the GenBank database. JS-A is represented by a triangle. The phylogenetic tree was constructed by the adjacency method in MEGA 7 (http://www.megasoftware.net). Bootstrap analysis was performed using 1,000 iterations and the bootstrap value was indicated on each branch. Scale bar indicates nucleotide substitution at each site.

To determine when PEDV IgG, IgA, and neutralizing antibodies were detected in infected piglets, PEDV IgG, IgA, and neutralizing antibodies were measured in serum samples collected at 7, 14, and $21 \mathrm{dpi}$. The OD values of serum $\operatorname{IgG}$ and $\operatorname{IgA}$ in piglets inoculated with PEDV gradually increased at 7, 14, and 21 dpi, and the antibody titer gradually increased and peaked at 


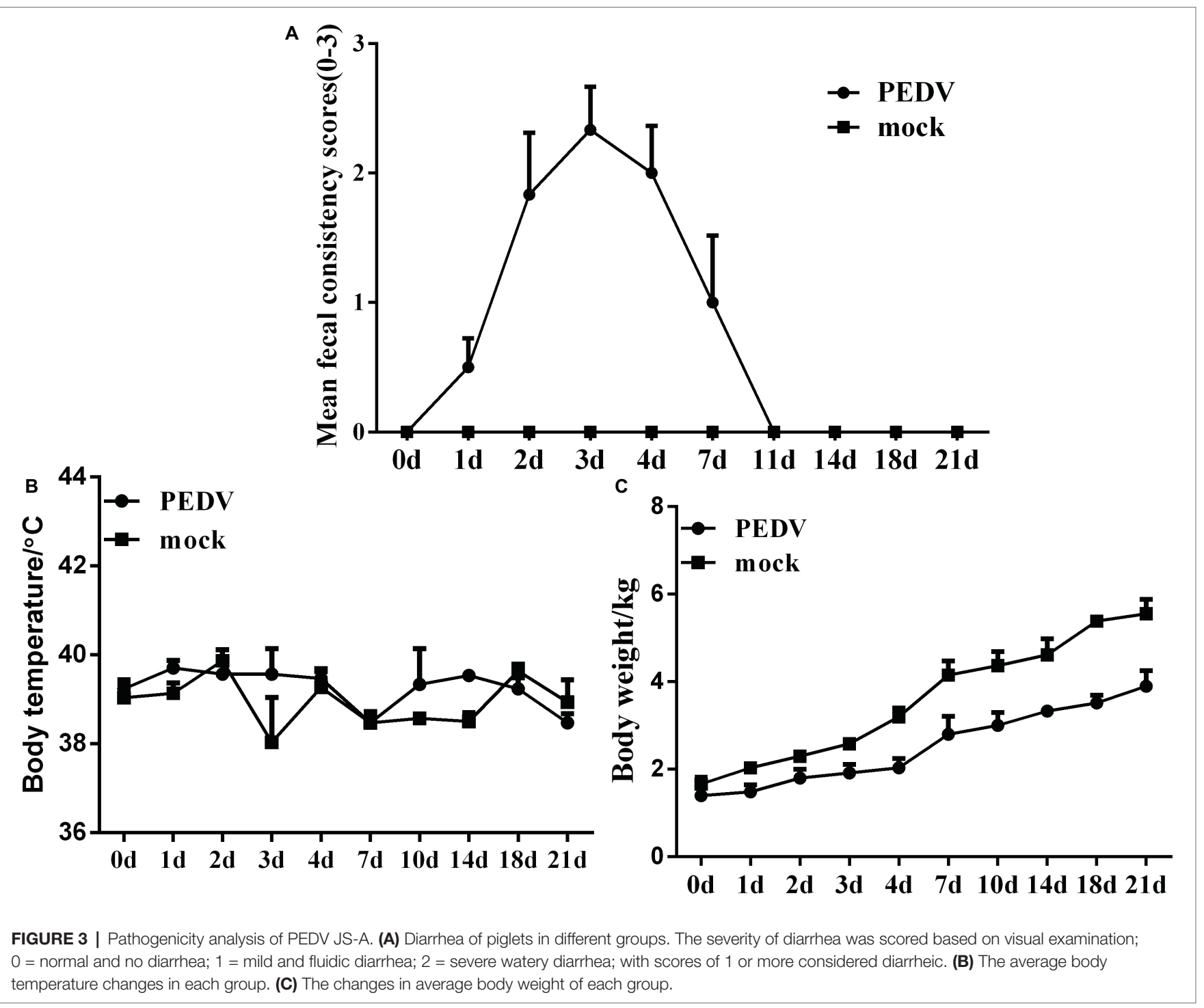

21 dpi (Figures 4B,C). Neutralizing antibodies in collected serum samples were detected, and it was found that the antibody titer gradually increased and reached a peak at $21 \mathrm{dpi}$, as high 1:255 (Figure 4D). PEDV IgG, IgA, and neutralizing antibodies were not observed in the serum of control piglets.

\section{Gross Pathology and Histopathology}

In order to ascertain changes in piglets infected with JS-A about the overall histology and pathology, three of six piglets in each group were randomly selected for autopsy at $4 \mathrm{dpi}$. The typical gross lesion seen in infected pigs is the accumulation of large amounts of yellow watery contents in the small intestine, resulting in thin and transparent intestinal wall and gas expansion due to atrophy of intestinal villi (Figure 5A). No other injuries were observed in any organs of the control pigs (Figure 5B). Histopathological examination showed necrosis of small intestinal epithelial cells, atrophy of intestinal villi and vacuolation in infected pigs compared with the control group (Figures 5C,D), but the intestines of the negative control were normal (Figures 5E,F).
Consistent with histopathological findings, PEDV antigen was found in the cytoplasm of infected atrophic intestinal villous epithelial cells (Figures 5G,H), and no PEDV IHC antigen was found in uninfected piglets (Figures 5I,J).

\section{Porcine Epidemic Diarrhea Virus JS-A Infection Suppresses Neonatal Fc Receptor Expression Through Down- Regulation of Toll-Like Receptors and Downstream Signaling Molecules}

At 3 dpi, PEDV JS-A infection downregulated TLR3, TLR4, TLR7, TLR8, and TLR9 expression, as compared to the control group. Further, the corresponding downstream molecules TRIF, MyD88 (subunits A and B), and TRAF6 were significantly downregulated, eventually leading to down-regulation of the NF- $\kappa \mathrm{B}$ pathway $(p<0.05$ ) (Figures $6 \mathrm{~A}, \mathrm{~B})$. However, at 21 dpi, TLR4, TLR7, and corresponding downstream MyD88 (subunits A and B) were up-regulated, further up-regulating NF- $\mathrm{BB}$. We found that FcRn expression was down-regulated 

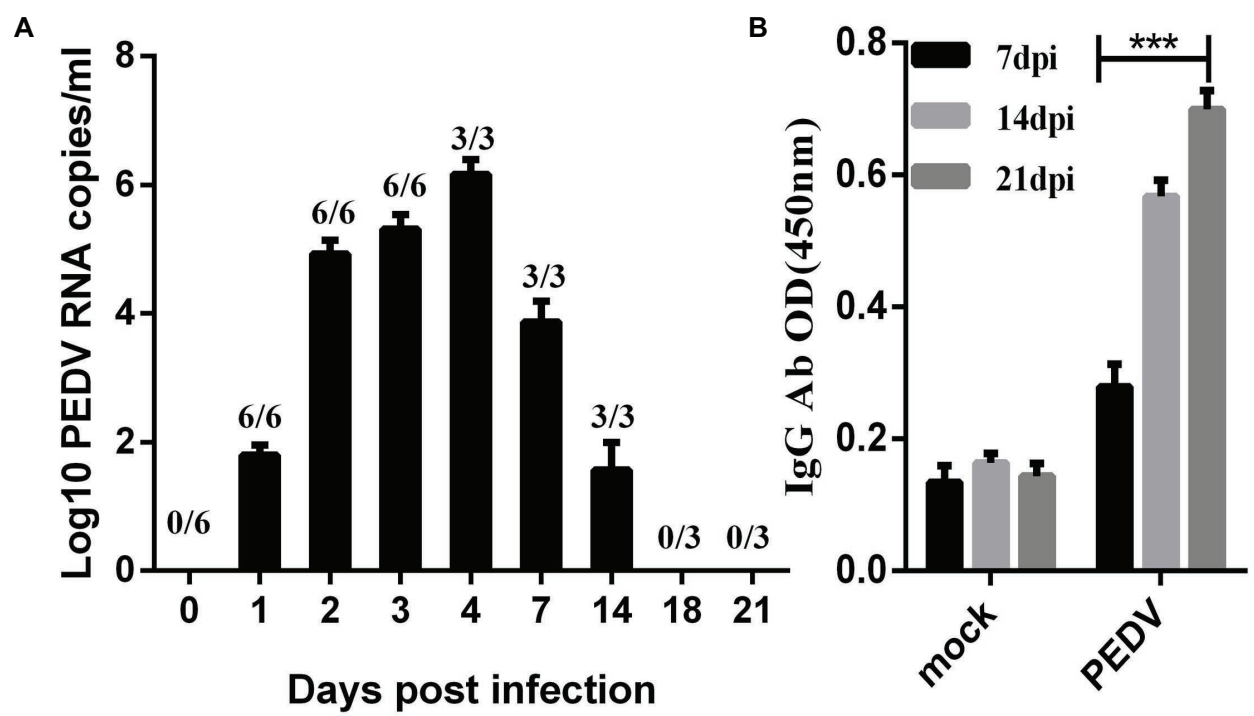

C

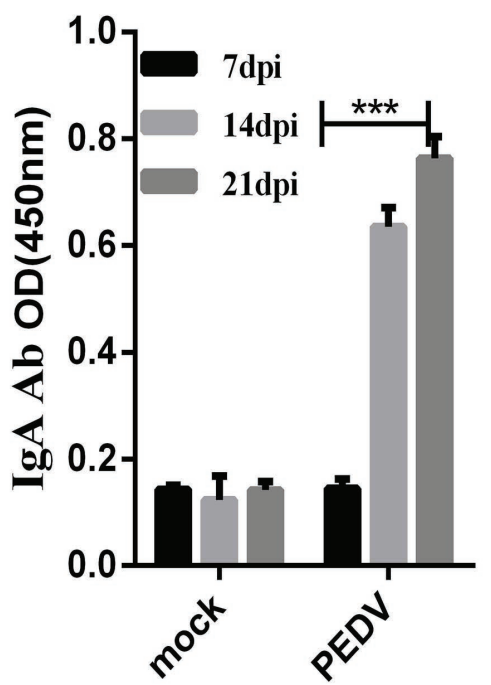

D

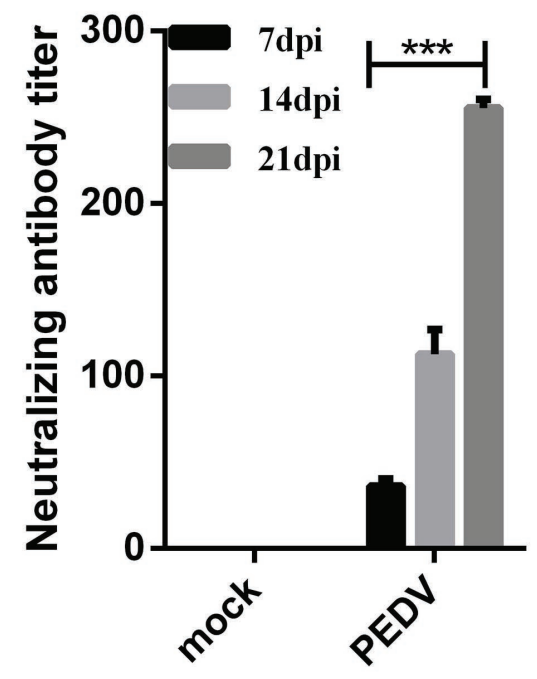

FIGURE 4 | Fecal detoxification and humoral immune response in piglets challenged with PEDV-JS-A. (A) Fecal virus shedding in PEDV-challenged piglets. (B) Detection of PEDV IgG in serum samples from PEDV-challenged piglets. (C) PEDV IgA detection in serum samples from PEDV-challenged piglets. (D) Detection of neutralizing antibodies in serum samples of immunized piglets. (Data were analyzed as mean \pm SEM) $(n=3$ for the JS-A group, and statistically significant for the unchallenged group by Student's t test. " $" p<0.001$ ).

at $4 \mathrm{dpi}$, yet up-regulated at $21 \mathrm{dpi}$, and the regulation of FcRn was closely related to regulation of NF- $\mathrm{BB}$ (Figures 6C,D).

\section{DISCUSSION}

Despite the use of commercially inactivated vaccines, the characteristics of PED infection and its epidemiology are highly significant, with morbidity and mortality rates approaching $100 \%$ in piglets (Pan et al., 2012). In 2013, a sudden PED outbreak occurred in the United States and spread quickly throughout the country, as well as Canada and Mexico, resulting in high mortality of newborn piglets and serious financial problems (Mole, 2013; Stevenson et al., 2013; Vlasova et al., 2014).

In this study, the isolated JS-A strain was more similar to the PEDV GD-1 strain, which was distant from classical strains such as CV777, LZC, and SM98, suggesting that PEDV is continuously evolving, with variation in the epidemiological process. Therefore, vaccines made from classical strains cannot provide effective protection, which is consistent with current findings. The PEDV S gene evolutionary tree and homology 

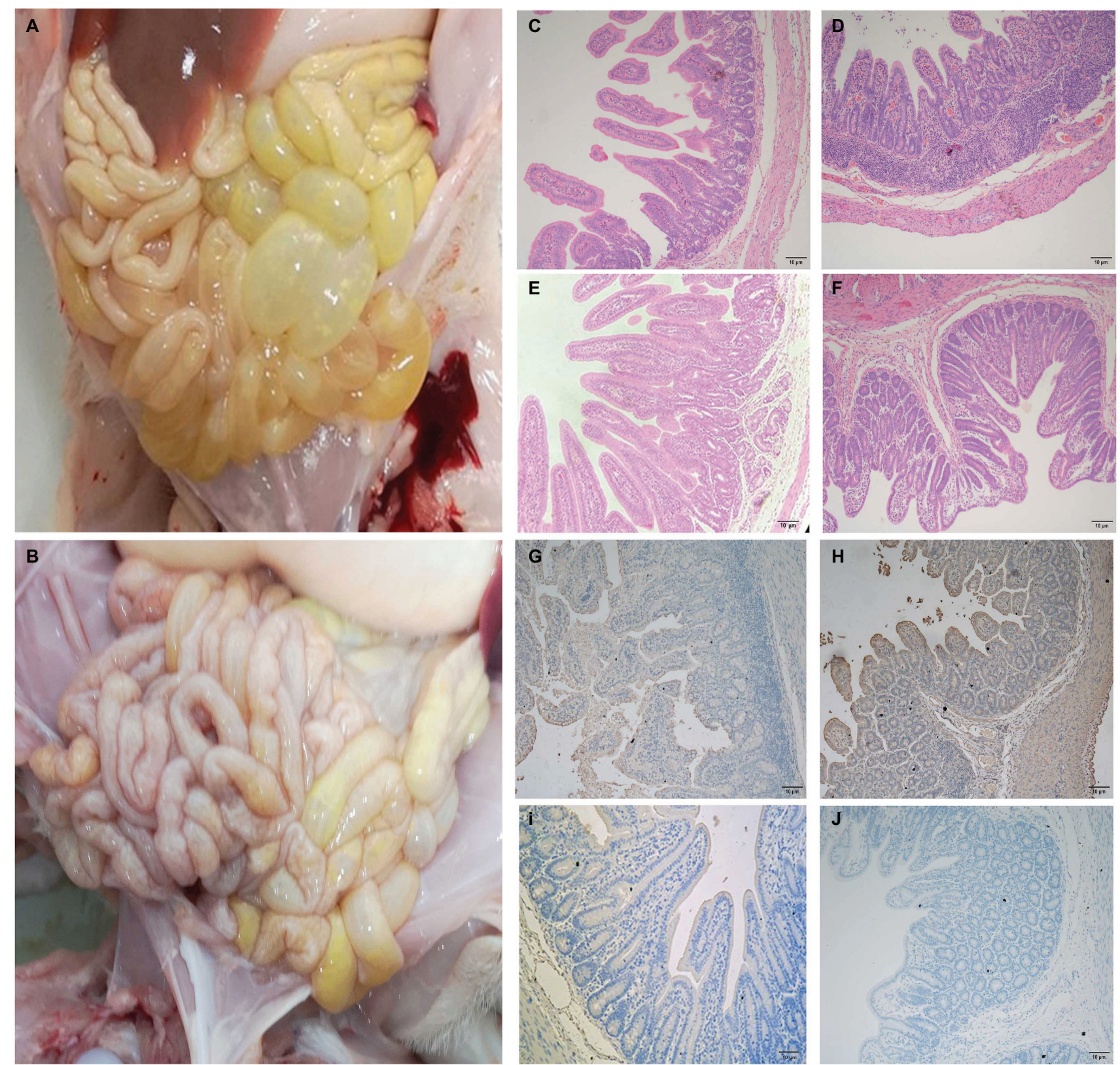

FIGURE 5 | The intestinal microscopic lesions of piglets. (A) Macroscopic damage of piglets challenged with PEDV at 4 dpi. (B) Macroscopic view of 4 dpi negative control piglets. (C,D) H\&E stained jejunal (C) and ileal (D) tissue sections of piglets challenged with PEDV. (E,F) H\&E stained jejunal (E) and ileal (F) tissue sections of negative control pigs. (G,H) PEDV challenge immunohistochemical staining of jejunal (G) and ileal (H) tissue sections of piglets. Immunohistochemically stained jejunal $\mathbf{( I )}$ and ileal $\mathbf{( J )}$ tissue sections of $\mathbf{( I , J )}$ negative control pigs.

were further analyzed. The phylogenetic tree was divided into two evolutionary branches, namely G1 and G2 groups. The G1 group contained classic strains such as CV777, DR13, and SM98. The G2 group has been the most prevalent strain since 2010. JS-A, which has been separated this time, also belongs to G2a. This shows that JS-A is indeed the dominant strain in current pig farms. Induced stronger primary and anamnestic immune responses. The JS-A strain belongs to the non-S-INDEL, which is highly pathogenic.
To study the pathogenicity of PEDV JS-A, 5-day-old piglets were inoculated orally. As expected, vomiting and severe diarrhea were observed in piglets from 1 to $6 \mathrm{dpi}$, indicating that JS-A was pathogenic to newborn piglets. Fecal swabs were collected from piglets infected with PEDV, and viral fecal shedding was detected by real-time PCR. PEDV RNA was detected from 1 to $14 \mathrm{dpi}$, suggesting that PEDV infection via fecal-oral contamination may be the main transmission route of piglet diarrhea in pig farms. Previously, it has been reported that coronavirus HKU15 

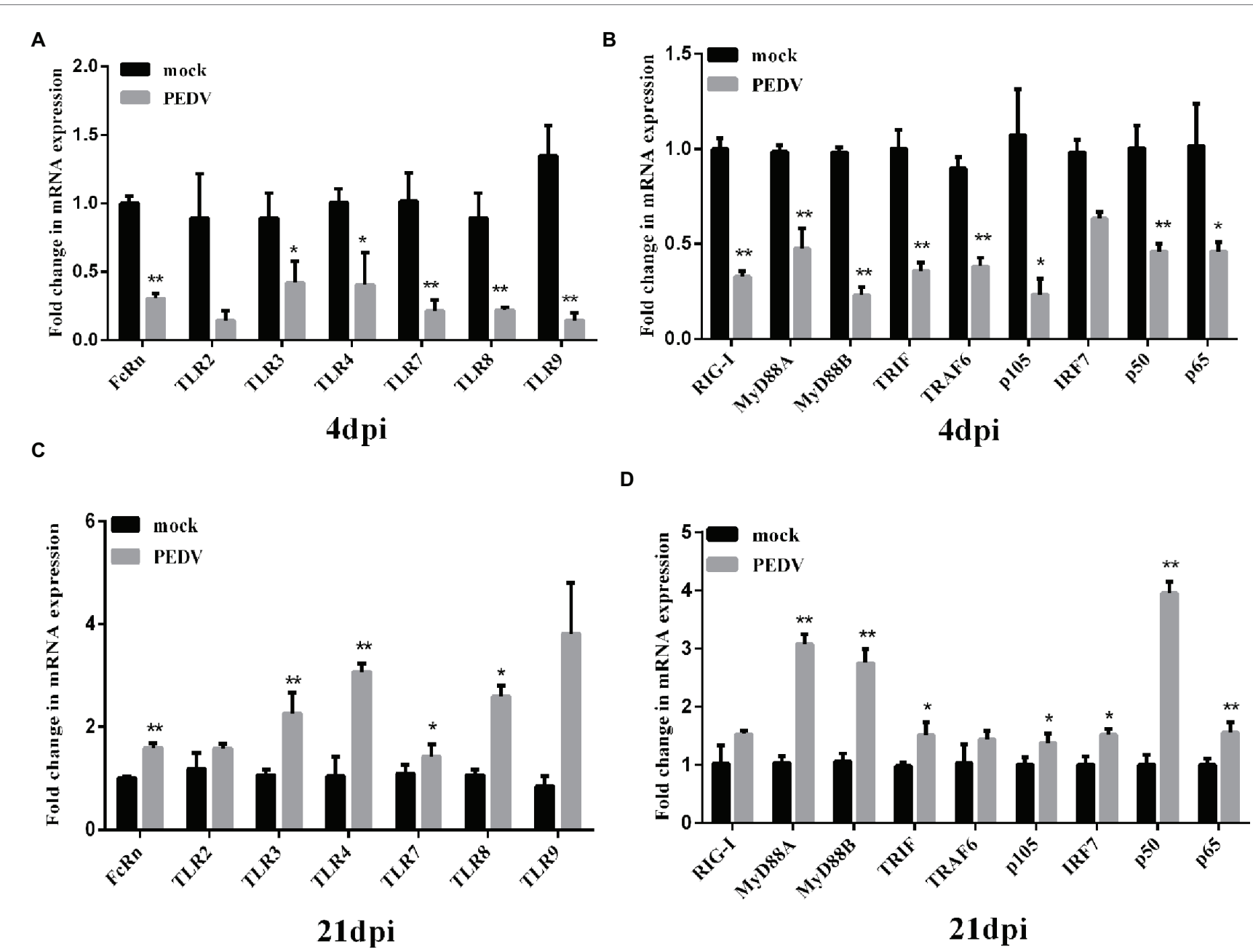

D

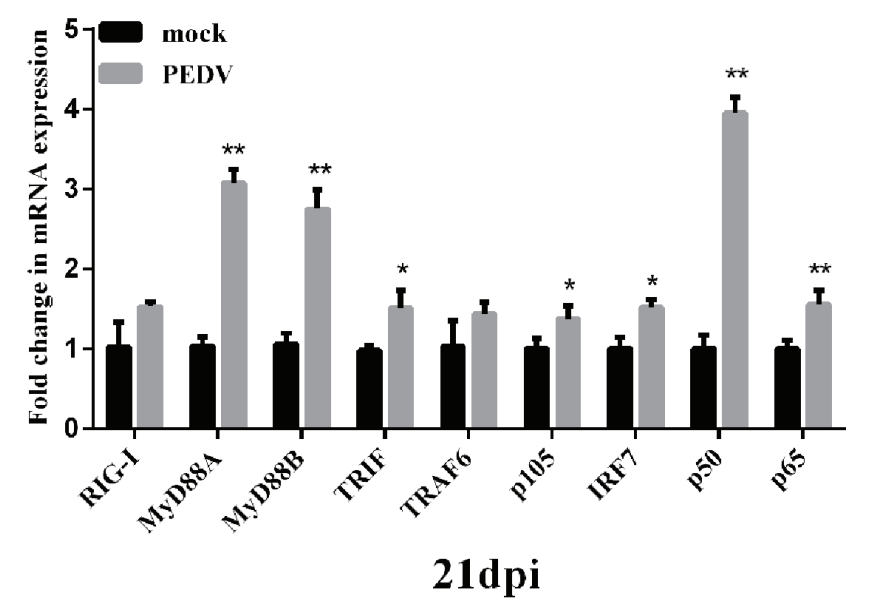

FIGURE 6 | Changes in FCRn, TLRs and its downstream-signaling molecules mRNA expression on intestinal mucosa of piglets induced by PEDV JS-A at 4 and 21 dpi. (A,C) The mRNA expressions of FcRn, TLR2, TLR3, TLR4, TLR7, TLR8, TLR9 at the intestinal mucosa from piglets after infection with JS-A at 4dpi (A) and $21 \mathrm{dpi}$ (C) by RT-qPCR. (B,D) The mRNA expressions of RIG-I, TRIF, MyD88A, MyD88B, IRF7, TRAF6, NF-kB1 (p105), NF-kB1 (p50), RelA (p65) at the intestinal mucosa from piglets after infection with JS-A at 4dpi (B) and 21dpi (D) by RT-qPCR. All samples were tested in triplicate and the results are expressed as fold changes relative to the control animals Data are presented as means \pm SEM. Significant difference between PEDV JS-A and control group are expressed with their $p . " p<0.05 ; " p<0.01$.

may be transmitted via the respiratory route, in addition to fecal-oral transmission (Woo et al., 2017; Li et al., 2018). Notably, we only observed microlesions in the jejunum and ileum of piglets infected with JS-A, but PEDV infection did not cause death of newborn piglets, despite drowsiness, anorexia, and watery diarrhea in these animals. These results suggest that PEDV JS-A has a milder toxicity and may be a weak strain. At the same time, there were slight differences in body weight and no significant differences in body temperature during infection.

We also tested the serum-specific IgG, IgA, and neutralizing antibody levels in piglets at 7,14, and $21 \mathrm{dpi}$ and found that the antibody levels of IgG and IgA gradually increased with time. Further, the serum IgA level was higher than that of IgG. PEDV-challenged protection during the first infection was positively correlated with IgA and IgG antibodies in intestinal-associated lymphoid tissues and blood (de Arriba et al., 2002), indicating that $\mathrm{VN}$ antibody can be detected in piglets inoculated with PEDV JS-A, and the titer of VN antibody gradually increases and remains high at the end of the experiment (Chen et al., 2016). In our study, PEDV VN antibody was detected as early as $7 \mathrm{dpi}$ in piglets infected with PEDV. The antibody titer of $\mathrm{VN}$ increased and remained high up to $21 \mathrm{dpi}$, at which point all pigs in the challenged group recovered completely.

In vitro studies have proven to be highly dependent on strains, and cell types cannot be used to assess innate immune responses against low virulence strains (Kint et al., 2015). PEDV has been reported to induce innate immune responses in vivo. However, few studies have been reported. Therefore, in this study, in vivo models were used to further observe the natural TLRs and signaling pathway homeostasis regulated by the gut microbiota. Natural immunity is the first line of host defense against a variety of pathogenic infections (Li et al., 2013). 
Furthermore, in vitro PEDV $\mathrm{N}$ and $\mathrm{E}$ protein mediated $\mathrm{NF}-\kappa \mathrm{B}$ activation and had effects on cell growth and ER stress, upregulating IL-8 expression (Xu et al., 2013; Cao et al., 2015b). However, PEDV non-S-INDEL $\mathrm{N}$ and nsp1 protein inhibited NF- $\kappa B$ activation (Ding et al., 2014; Zhang et al., 2017). MyD88 is an essential component of the innate immune response to SARS-CoV infection in mice in vivo (Totura et al., 2015). The pathogenesis of PEDV is strain specific. The lack of TRAF6 leads to enhanced viral replication and a significant reduction in the production of type I IFN after infection with RNA virus. In vivo, PEDV non-S-INDEL down-regulates the NF- $\kappa \mathrm{B}$ signaling pathway through negative regulation of TLR4, TLR7, TLR8, and TLR9, resulting in the final attenuation of MyD88, TRIF, and TRAF6 gene expression (Temeeyasen et al., 2018).

The IgA plays a major role in the mucosal anti-PEDV infection immunity. However, recent studies have found that IgG also plays an important role against pathogen infection in mucosal sites, while the neonatal $\mathrm{Fc}$ receptor $(\mathrm{FcRn})$ is the only $\operatorname{IgG}$ transport receptor (Tsuruta et al., 2012). Down-regulation of FcRn and pIgR has been shown in the tracheal mucosa of SHIV/ SIV-infected rhesus macaques (Wang and Yang, 2016; Li et al., 2017). Further, transmissible gastroenteritis virus infection up-regulates FcRn through TLR3 and RIG-I in porcine intestinal epithelial cells (unpublished results). PEDV JS-A can down-regulate NF- $\kappa \mathrm{B}$ signaling pathway by inhibiting mRNA expression of TLR3, TLR4, TLR7, TLR8, and TLR9 at $4 \mathrm{dpi}$, resulting in the final attenuation of FcRn gene expression. In contrast, FcRn expression was up-regulated through TLR3, TLR4, TLR8 at $21 \mathrm{dpi}$.

In conclusion, we successfully isolated the PEDV JS-A strain, and phylogenetic analysis indicated that the major parent of JS-A strain was identified as strain FL2013 or GD-1. The study results showed that JS-A was a variant PEDV strain with weak pathology to piglets, compared to other emerging strains. High levels of serum IgG, IgA, and VN antibodies were also detected in the sera of infected piglets at $21 \mathrm{dpi}$. In addition, inoculation of piglets with JS-A suppressed FcRn expression via TLR,

\section{REFERENCES}

Brian, D. A., and Baric, R. S. (2005). Coronavirus genome structure and replication. Curr. Top. Microbiol. Immunol. 287, 1-30. doi: 10.1007/3-540-26765-4_1

Cao, L., Ge, X., Gao, Y., Herrler, G., Ren, Y., Ren, X., et al. (2015b). Porcine epidemic diarrhea virus inhibits dsRNA-induced interferon- $\beta$ production in porcine intestinal epithelial cells by blockade of the RIG-I-mediated pathway. Virol. J. 12:127. doi: 10.1186/s12985-015-0345-x

Cao, L., Ge, X., Gao, Y., Ren, Y., Ren, X., and Li, G. (2015a). Porcine epidemic diarrhea virus infection induces NF-kappaB activation through the TLR2, TLR3 and TLR9 pathways in porcine intestinal epithelial cells. J. Gen. Virol. 96, 1757-1767. doi: 10.1099/vir.0.000133

Chen, Q., Li, G., Stasko, J., Thomas, J. T., Stensland, W. R., Pillatzki, A. E., et al. (2014). Isolation and characterization of porcine epidemic diarrhea viruses associated with the 2013 disease outbreak among swine in the United States. J. Clin. Microbiol. 52, 234-243. doi: 10.1128/JCM.02820-13

Chen, J., Liu, X., Shi, D., Shi, H., Zhang, X., and Feng, L. (2012). Complete genome sequence of a porcine epidemic diarrhea virus variant. J. Virol. 86:3408. doi: 10.1128/JVI.07150-11

Chen, Q., Thomas, J. T., Gimenez-Lirola, L. G., Hardham, J. M., Gao, Q., Gerber, P. F., et al. (2016). Evaluation of serological cross-reactivity and cross-neutralization between the United States porcine epidemic diarrhea
RIG-I, and NF- $\mathrm{BB}$ mRNA expressions at $4 \mathrm{dpi}$, yet induced FcRn expression at $21 \mathrm{dpi}$ in infected intestinal mucosa. All results showed that innate immunity was suppressed at $4 \mathrm{dpi}$ and that innate immunity might have been activated by the virus at $21 \mathrm{dpi}$. The down-regulated expression of FcRn resulted in its incapacity to transport antibodies, providing another insight into the immune escape strategy of PEDV.

\section{DATA AVAILABILITY STATEMENT}

The datasets generated for this study can be found in the PEDV JS-A (GenBank: MH748550.1).

\section{ETHICS STATEMENT}

The animal study was reviewed and approved by the Scientific Ethic Committee of Huazhong Agricultural University; Huazhong Agricultural University. Written informed consent was obtained from the owners for the participation of their animals in this study.

\section{AUTHOR CONTRIBUTIONS}

SQ and ZL wrote the paper and conceived and initiated the study. SQ, WZ, and XJ extracted the data set. SQ, WZ, XJ, $\mathrm{ZS}, \mathrm{YZ}$, and YX performed the analysis. All authors reviewed the manuscript.

\section{FUNDING}

This work was supported by the National Natural Science Foundation of China (31572500 to ZL). virus prototype and S-INDEL-variant strains. BMC Vet. Res. 12:70. doi: 10.1186/s12917-016-0697-5

de Arriba, M. L., Carvajal, A., Pozo, J., and Rubio, P. (2002). Isotype-specific antibody-secreting cells in systemic and mucosal associated lymphoid tissues and antibody responses in serum of conventional pigs inoculated with PEDV. Vet. Immunol. Immunopathol. 84, 1-16. doi: 10.1016/ S0165-2427(01)00386-5

DeDiego, M. L., Nieto-Torres, J. L., Regla-Nava, J. A., Jimenez-Guardeno, J. M., Fernandez-Delgado, R., Fett, C., et al. (2014). Inhibition of NF-kappaBmediated inflammation in severe acute respiratory syndrome coronavirusinfected mice increases survival. J. Virol. 88, 913-924. doi: 10.1128/JVI.02576-13

Ding, Z., Fang, L., Jing, H., Zeng, S., Wang, D., Liu, L., et al. (2014). Porcine epidemic diarrhea virus nucleocapsid protein antagonizes beta interferon production by sequestering the interaction between IRF3 and TBK1. J. Virol. 88, 8936-8945. doi: 10.1128/JVI.00700-14

Guo, J., Li, F., He, Q., Jin, H., Liu, M., Li, S., et al. (2016b). Neonatal fc receptor-mediated IgG transport across porcine intestinal epithelial cells: potentially provide the mucosal protection. DNA Cell Biol. 35, 301-309. doi: 10.1089/dna.2015.3165

Guo, J., Li, F., Qian, S., Bi, D., He, Q., Jin, H., et al. (2016a). TGEV infection up-regulates FcRn expression via activation of NF-kappaB signaling. Sci. Rep. 6:32154. doi: 10.1038/srep32154 
Huang, Y. W., Dickerman, A. W., Pineyro, P., Li, L., Fang, L., Kiehne, R., et al. (2013). Origin, evolution, and genotyping of emergent porcine epidemic diarrhea virus strains in the United States. MBio 4, e00737-e00713. doi: $10.1128 / \mathrm{mBio} .00737-13$

Kint, J., Fernandez-Gutierrez, M., Maier, H. J., Britton, P., Langereis, M. A., Koumans, J., et al. (2015). Activation of the chicken type I interferon response by infectious bronchitis coronavirus. J. Virol. 89, 1156-1167. doi: 10.1128/ JVI.02671-14

Lee, S., Kim, Y., and Lee, C. (2015). Isolation and characterization of a Korean porcine epidemic diarrhea virus strain KNU-141112. Virus Res. 208, 215-224. doi: 10.1016/j.virusres.2015.07.010

Lee, D. K., Park, C. K., Kim, S. H., and Lee, C. (2010). Heterogeneity in spike protein genes of porcine epidemic diarrhea viruses isolated in Korea. Virus Res. 149, 175-182. doi: 10.1016/j.virusres.2010.01.015

Li, B., Liu, H., He, K., Guo, R., Ni, Y., Du, L., et al. (2013). Complete genome sequence of a recombinant porcine epidemic diarrhea virus strain from eastern China. Genome Announc. 1:e0010513. doi: 10.1128/genomeA.00105-13

Li, D., Wang, F. J., Yu, L., Yao, W. R., Cui, Y. F., and Yang, G. B. (2017). Expression of pIgR in the tracheal mucosa of SHIV/SIV-infected rhesus macaques. Zool. Res. 38, 44-48. doi: 10.13918/j.issn.2095-8137.2017.007

Li, Y., Wu, Q., Huang, L., Yuan, C., Wang, J., and Yang, Q. (2018). An alternative pathway of enteric PEDV dissemination from nasal cavity to intestinal mucosa in swine. Nat. Commun. 9:3811. doi: 10.1038/s41467-018-06056-w

Mole, B. (2013). Deadly pig virus slips through US borders. Nature 499:388. doi: $10.1038 / 499388 \mathrm{a}$

Moynagh, P. N. (2005). The NF-kappaB pathway. J. Cell Sci. 118, 4589-4592. doi: $10.1242 /$ jcs.02579

Oh, J., Lee, K. W., Choi, H. W., and Lee, C. (2014). Immunogenicity and protective efficacy of recombinant S1 domain of the porcine epidemic diarrhea virus spike protein. Arch. Virol. 159, 2977-2987. doi: 10.1007/s00705-014-2163-7

Pan, Y., Tian, X., Li, W., Zhou, Q., Wang, D., Bi, Y., et al. (2012). Isolation and characterization of a variant porcine epidemic diarrhea virus in China. Virol. J. 9:195. doi: 10.1186/1743-422X-9-195

Pyzik, M., Rath, T., Kuo, T. T., Win, S., Baker, K., Hubbard, J. J., et al. (2017). Hepatic FcRn regulates albumin homeostasis and susceptibility to liver injury. Proc. Natl. Acad. Sci. USA 114, E2862-E2871. doi: 10.1073/pnas.1618291114

Shan, Y., Liu, Z. Q., Li, G. W., Chen, C., Luo, H., Liu, Y. J., et al. (2018). Nucleocapsid protein from porcine epidemic diarrhea virus isolates can antagonize interferon-lambda production by blocking the nuclear factorkappaB nuclear translocation. J. Zhejiang Univ. Sci. B 19, 570-580. doi: 10.1631/jzus.B1700283

Song, D., and Park, B. (2012). Porcine epidemic diarrhoea virus: a comprehensive review of molecular epidemiology, diagnosis, and vaccines. Virus Genes 44, 167-175. doi: 10.1007/s11262-012-0713-1

Stevenson, G. W., Hoang, H., Schwartz, K. J., Burrough, E. R., Sun, D., Madson, D., et al. (2013). Emergence of porcine epidemic diarrhea virus in the United States: clinical signs, lesions, and viral genomic sequences. J. Vet. Diagn. Investig. 25, 649-654. doi: 10.1177/1040638713501675

Subramaniam, S., Yugo, D. M., Heffron, C. L., Rogers, A. J., Sooryanarain, H., LeRoith, T., et al. (2018). Vaccination of sows with a dendritic cell-targeted porcine epidemic diarrhea virus $S 1$ protein-based candidate vaccine reduced viral shedding but exacerbated gross pathological lesions in suckling neonatal piglets. J. Gen. Virol. 99, 230-239. doi: 10.1099/jgv.0.001001
Temeeyasen, G., Sinha, A., Gimenez-Lirola, L. G., Zhang, J. Q., and Piñeyro, P. E. (2018). Differential gene modulation of pattern-recognition receptor TLR and RIG-I-like and downstream mediators on intestinal mucosa of pigs infected with PEDV non S-INDEL and PEDV S-INDEL strains. Virology 517, 188-198. doi: 10.1016/j.virol.2017.11.024

Totura, A. L., Whitmore, A., Agnihothram, S., Schäfer, A., Katze, M. G., Heise, M. T., et al. (2015). Toll-like receptor 3 signaling via TRIF contributes to a protective innate immune response to severe acute respiratory syndrome coronavirus infection. MBio 6, e00638-e00615. doi: 10.1128/mBio.00638-15

Tsuruta, T., Inoue, R., Tsukahara, T., Nakamoto, M., Hara, H., Ushida, K., et al. (2012). Commensal bacteria coated by secretory immunoglobulin A and immunoglobulin $\mathrm{G}$ in the gastrointestinal tract of pigs and calves. Anim. Sci. J. 83, 799-804. doi: 10.1111/j.1740-0929.2012.01026.x

Vlasova, A. N., Marthaler, D., Wang, Q., Culhane, M. R., Rossow, K. D., Rovira, A., et al. (2014). Distinct characteristics and complex evolution of PEDV strains, North America, May 2013-February 2014. Emerg. Infect. Dis. 20, 1620-1628. doi: 10.3201/eid2010.140491

Wang, L., Hayes, J., Byrum, B., and Zhang, Y. (2016). US variant porcine epidemic diarrhea virus: histological lesions and genetic characterization. Virus Genes 52, 578-581. doi: 10.1007/s11262-016-1334-x

Wang, Y., and Yang, G. B. (2016). Alteration of polymeric immunoglobulin receptor and neonatal fc receptor expression in the gut mucosa of immunodeficiency virus-infected rhesus macaques. Scand. J. Immunol. 83, 235-243. doi: 10.1111/sji.12416

Woo, P. C., Lau, S. K., Tsang, C. C., Lau, C. C., Wong, P. C., Chow, F. W., et al. (2017). Coronavirus HKU15 in respiratory tract of pigs and first discovery of coronavirus quasispecies in 5'-untranslated region. Emerg. Microbes Infect. 6:e53. doi: 10.1038/emi.2017.37

Wood, E. N. (1977). An apparently new syndrome of porcine epidemic diarrhoea. Vet. Rec. 100, 243-244. doi: 10.1136/vr.100.12.243

Xu, X., Zhang, H., Zhang, Q., Dong, J., Liang, Y., Huang, Y., et al. (2013). Porcine epidemic diarrhea virus $\mathrm{E}$ protein causes endoplasmic reticulum stress and up-regulates interleukin-8 expression. Virol. J. 10:26. doi: 10.1186/1743-422X-10-26

Yamamoto, R., Soma, J., Nakanishi, M., Yamaguchi, R., and Niinuma, S. (2015). Isolation and experimental inoculation of an S INDEL strain of porcine epidemic diarrhea virus in Japan. Res. Vet. Sci. 103, 103-106. doi: 10.1016/j. rvsc.2015.09.024

Zhang, Q., Liu, X., Fang, Y., Zhou, P., Wang, Y., and Zhang, Y. (2017). Detection and phylogenetic analyses of spike genes in porcine epidemic diarrhea virus strains circulating in China in 2016-2017. Virol. J. 14:194. doi: 10.1186/ s12985-017-0860-Z

Conflict of Interest: The authors declare that the research was conducted in the absence of any commercial or financial relationships that could be construed as a potential conflict of interest.

Copyright (c) 2019 Qian, Zhang, Jia, Sun, Zhang, Xiao and Li. This is an openaccess article distributed under the terms of the Creative Commons Attribution License (CC BY). The use, distribution or reproduction in other forums is permitted, provided the original author(s) and the copyright owner(s) are credited and that the original publication in this journal is cited, in accordance with accepted academic practice. No use, distribution or reproduction is permitted which does not comply with these terms. 